\title{
DEEP NEURAL NETWORKS FOR ABOVE-GROUND DETECTION IN VERY HIGH SPATIAL RESOLUTION DIGITAL ELEVATION MODELS
}

\author{
D. Marmanis ${ }^{a, b}$, F. Adam ${ }^{a}$, M. Datcu ${ }^{a}$, T. Esch ${ }^{a}$, U. Stilla $^{b}$ \\ ${ }^{a}$ EOC, German Aerospace Center, Wessling, Germany - (Dimitrios. Marmanis, Fathalrahman.Adam, \\ Mihai.Datcu, Thomas.Esch)@dlr.de \\ ${ }^{\mathrm{b}}$ Chair of Photogrammetry \& Remote Sensing, Technische Universitaet Muenchen, Germany - stilla@ tum.de
}

Commission III, WG III/7

KEY WORDS: Deep Learning, Multilayer Perceptrons, Ground Filtering, DEM, Classification

\begin{abstract}
:
Deep Learning techniques have lately received increased attention for achieving state-of-the-art results in many classification problems, including various vision tasks. In this work, we implement a Deep Learning technique for classifying above-ground objects within urban environments by using a Multilayer Perceptron model and VHSR DEM data. In this context, we propose a novel method called M-ramp which significantly improves the classifier's estimations by neglecting artefacts, minimizing convergence time and improving overall accuracy. We support the importance of using the M-ramp model in DEM classification by conducting a set of experiments with both quantitative and qualitative results. Precisely, we initially train our algorithm with random DEM tiles and their respective point-labels, considering less than $0.1 \%$ over the test area, depicting the city center of Munich $\left(25 \mathrm{~km}^{2}\right)$. Furthermore with no additional training, we classify two much larger unseen extents of the greater Munich area $\left(424 \mathrm{~km}^{2}\right)$ and Dongying city, China $\left(257 \mathrm{~km}^{2}\right)$ and evaluate their respective results for proving knowledge-transferability. Through the use of Mramp, we were able to accelerate the convergence by a magnitude of 8 and achieve a decrease in above-ground relative error by $24.8 \%$ and $5.5 \%$ over the different datasets.
\end{abstract}

\section{INTRODUCTION}

\subsection{Previous Work}

Building and ground detection is a crucial step for many applications in the analysis of Digital Elevation Models (DEM) such as urban monitoring, real estate, disaster response and 3D city reconstruction. Automatic methods that can robustly detect above-ground objects in such context could prove very valuable as extended human labour and the large cost involved could be omitted. In the literature there is a plethora of such proposed methods where different techniques and input data are considered. Belli et al. (2001) proposed a method to extract and model above ground objects by estimating the Digital Terrain Model (DTM) from a DEM obtained from stereoscopic aerial colour images. The ground surface is estimated through the use of $2 \mathrm{D}$ harmonics and Fourier series. With respect to this framework, above-ground objects are considered outliers. Zingaretti et al. (2007) proposed a method using raw LIDAR data where a boosted decision tree-classifier was used to identify optimal classification rules and separate data in buildings, ground, and vegetation classes when applied over segmented data. Hao et al. (2009) was able to accurately detect the buildings using a combination of filtering, clustering, and thresholding over a LIDAR dataset. Baillard and Maitre (1999) used a stereopair of images to initially compute the DEM model and then segmented the above-ground objects using a Markov Random Field model. Afterwards, they differentiated between vegetation and buildings through the use of radiometric analysis. Ortner et al. (2007) proposed a method for directly extracting vectorial land registers from DEM data using an energy minimization model, where the assumption that buildings are composed by simple rectangles is considered.
Furthermore, this method was improved by Tournaire et al. (2010), so it can process data more efficiently.

Despite the extended work in the field, above-ground and building detection still remains an open research area. In our view, algorithms that make strong assumptions upon the size or shape of the high-structures in the data are unlikely to robustly detect above-ground structures in different urban scenes. Therefore, our goal in this work is to construct a generic framework, without making presumptions upon the size, shape, extent or resolution of the input DEM data.

\subsection{Deep Neural Networks for Above-Ground Detection}

In this work, we investigate the potential of Deep Neural Network (DNN) systems in above-ground object detection within urban environments. Specifically, our target is to separate the high-standing structures (trees and building) from their surrounding terrain over large data extents with highaccuracy. To achieve this goal we construct a generic algorithm based on Deep Neural Networks and argue that such a model is capable for detecting above-ground objects over unseen areas, even when data are generated using different sensors. To support our claim we conduct a series of experiments using two very heterogeneous DEMs acquired by different sensors with different resolutions.

The rest of this work is organized as follows. Chapter 2 analyses the DNN algorithm in detail. Chapter 3 discusses the algorithms and workflow processes used for above-ground object detection. Chapter 4 and 5 deal with the description of test datasets and their experimental results. We end this work in Chapter 6 where we discuss our results and summarize our contributions. 


\section{THE DEEP LEARNING ALGORITHM}

\subsection{Deep Neural Networks}

Deep Neural Networks is a sub-field of Deep Learning (DL) expressed by ordinary Artificial Neural Networks with multiple hidden-layers. These multiprocessing stage distributed architectures allow the system to learn hierarchical representations by combining features of different abstraction levels generated by the various layers. The key element of such Deep Learning systems is the projection of their input data into a new space through the use of a non-linear function on each processing stage (hidden-layer). Via such a procedure, DNNs are capable of effectively extracting features over highdimensional structured data and achieve high performance in various classification tasks.

\subsection{Multilayer Perceptrons for Above-Ground Object Detection}

Probably the most broadly known architecture in the context of Artificial Neural Networks is the Multilayer Perceptron algorithm (MLP) which is a discriminative, supervised classification and regression model trained through standard Backpropagation (Rumelhart et al., 1986). Furthermore, MLPs are fully connected networks which mean that they relate each and every variable of a hidden-layer to all variables in the proceeding layer. Interestingly, MLPs are a modification of the standard Linear Perceptrons, introduced by Rosenblatt in the 1950s (Rosenblatt, 1957)

Despite their overall simplicity when compared to more complex and statistically promising Deep Leaning architectures, (e.g. Deep Belief Networks, Convolutional Neural Networks, etc.) with respect to our experiments, MLPs formulate a very concrete framework for accurately classifying large amounts of remotely sensed data, such as above-ground objects. Furthermore, their computational simplicity allowed us to conduct a very large number of different experiments that significantly improved our results and strengthened our understanding over these methods. Considering all these aspects, we concluded that it should suffice to focus on MLPs for conducting all our experiments, keeping in mind that other DL architectures may result in quantitatively better outcomes.

\subsection{Deep Multilayer Perceptron}

As long as Artificial Neural Networks are implemented using a single hidden layer, as commonly found in most of the software packages (e.g. MATLAB Neural Networks toolbox), the user has limited choices concerning the network's architecture, mainly selecting the number of hidden nodes or different optimization algorithms. However when one decides upon a deep architecture, finding an ideal local minimum in the error surface becomes a much harder task for the algorithm due to multiple non-linearities. Therefore in DL, it's essential for one to understand the individual components forming the neural network and then decide upon different techniques for achieving a good classification performance. Below, we refer to these individual parts which form our MLP model.

\subsection{Layer Architecture}

Our MLP is composed by 8 layers where layer- 1 is the input, layer- 8 is the output (Softmax Classifier) and the rest are hidden non-linear layers. The intuition lying behind this architecture is that the MLP model will be able to construct high level features, allowing it to adequately generalize over unseen data, even when trained with very few training data.
Formally, a MLP is a function $h$, that maps an input vector $x$, to a label vector $y$, through a set of non-linear functions $f$, with parameters $W$ and $b$ (weights and bias terms). Concretely, a single-layer neural network is expressed mathematically as:

$$
h_{W, b}(x)=f\left(\sum_{i} W_{i} x_{i}+b\right)
$$

2.4.1 ReLU Activation Function: One option for the nonlinear activation function in a deep MLP network is the Rectified-Linear Unit function (ReLU) which has some strong advantages towards the most frequently used sigmoid and hyperbolic-tangent activations. Specifically ReLU is preferable due to its sparse activation properties, it does not saturate or suffer from the vanishing gradient problem and it's computationally more efficient with respect to construction of large networks. The rectified linear function is denoted by:

$$
f(x)=\max (x, 0)
$$

\subsection{Learning the Weights - Model Optimization}

Our classification algorithm is trained by adjusting its weights using the Backpropagation algorithm (Rumelhart et al., 1986) with mini-batch gradient descent and a standard quadratic cost function for penalizing over misclassified examples.

\subsection{Regularization}

One weakness that all neural networks exhibit is their tendency to strongly overfit the training data. Therefore, in order to prevent this from occurring, multiple regularization techniques have to be employed, as suggested by the literature. Due to our limited training data, our model is very venerable to such overfitting phenomena therefore regularization becomes a crucial issue. Below we briefly present all regularization methods used in this study.

2.6.1 Weight Decay: Weight Decay (namely L2 regularization) is a common choice for including an additional term to the loss function by which large weights are penalized more, hence avoided by the system. The effect is that eventually the weights converge to smaller absolute values that would have done otherwise. Below, the loss-function including a weight-decay term is shown:

$$
E(W, b)=\frac{1}{2 m} \sum_{i=1}^{m}\left\|y_{i}-h\left(x_{i} ; W, b\right)\right\|^{2}+\frac{\lambda}{2} \sum_{l=1}^{L-1} W_{l}^{2}
$$

Where $m$ is the total number of training examples, $L$ denotes the respective layer in the network and $\lambda$ is the weight decay coefficient.

2.6.2 Momentum: A powerful method to avoid local minima and speed-up convergence is to use yet another regularization method, called momentum. Accordingly, by adding a momentum term in the loss function, one can avoid fluctuations between the weight-updates through the epochs during training. This is implemented by partially considering the effect of the previous weight update to the current gradient computation (epoch). Mathematically this is expressed as:

$$
\Delta W(t+1)=W-\alpha \frac{\partial E}{\partial W}+\beta \Delta W(t)-\lambda W(t)
$$


Where parameter $t$ denotes the epochs, $W$ the weight matrix, $E$ the loss-function and $\alpha, \beta \lambda$ are the learning rate, momentum and weight-decays coefficients respectively.

2.6.3 Max-norm: Even though momentum can be a powerful technique for speeding up convergence and helping avoid local minima, this can cause the weight to grow very large (Srebro et al., 2005). A good solution for this problem is given by Maxnorm regularization which constrains the sum of all weights connected to a unit to be smaller than a pre-defined constant. This can be mathematically expressed as:

$$
N(W)=\min \left(\left(\sum_{j} W_{i, j}^{(L)}\right), \mu\right)
$$

Where $i$ denotes a particular unit in the network, $j$ a certain weight-connection and $L$ the respective layer. The maximum allowed norm-value is defined by the $\mu$ variable. Typically, good values for constraining the max-norm are within the range of 3 and 4.

2.6.4 Dropout: Using a large number of units on each hidden layer is considered an advantage as the system learns very "powerful" representations, however to avoid extensive overfitting one should also employ a regularization technique called 'Dropout'. This method stochastically removes a percentage of the initial network units and their respective connecting-weights and can be seen as training an exponential number of "thinned" networks which extensively share the same weights (Srivastava et al., 2014). So for a neural network with $n$ units, dropout actually implements a collection of $2^{n}$ thinned neural networks with $O\left(n^{2}\right)$ or less number of units. Within this framework, one can then train very large networks without the concern of overfitting.

\subsection{Network Hyperparameter Random Search}

Finding a good set of hyperparameters for training an MLP model is probably one of the toughest aspects when one considers DL. Since there isn't any concrete rule for defining them, one can implement either a brute-force method (gridsearch) or a random-search within a predefined range of values. For standardizing our set of parameters, we used a random search method and the best values found over a validation dataset were used throughout our experiments. These values are listed in the Table 1 below.

\begin{tabular}{|c|c|}
\hline Hyperparameters & Value \\
\hline Dimension hidden h1 & 550 nodes \\
Dimension hidden h2 & 500 nodes \\
Dimension hidden h3 & 400 nodes \\
Dimension hidden h4 & 350 nodes \\
Dimension hidden h5 & 300 nodes \\
Dimension hidden h6 & Size of input vector \\
Drop-out probability input & $72 \%$ \\
Drop-out probability h1 & $57 \%$ \\
Drop-out probability h2 & $57 \%$ \\
Drop-out probability h3 & $55 \%$ \\
Drop-out probability h4 & $54 \%$ \\
Drop-out probability h5 & $54 \%$ \\
Weight Decay & 0.001635 \\
Initial Momentum & 0.256 \\
Final Momentum & 0.5759 \\
Start Epoch Momentum & 5 \\
Saturate Momentum Epoch & 38 \\
Learning Rate & 0.244 \\
\hline
\end{tabular}

Table 1. Standardized MLP hyperparameters

\section{METHODOLOGY}

\subsection{Processing Steps}

This section presents all the individual steps of our workflow process. Specifically, methods for merging and enhancing individual prediction are discussed in details, in addition to a novel method presented for improving overall accuracy and model convergence. The complete processing steps of our method are summarized in the diagram of Figure 2.

\subsection{Improving Classification with M-ramp}

Inspired by the idea that DNNs construct better feature representations when provided with an artificial destructed input (Vincent et al., 2008), we expanded this concept by artificially transforming the elevation of our input DEM model to partially mimic this behaviour. More precisely, by introducing intense artificial relief to the initial elevation model, we are forcing the MLP to detect strong dependencies and patterns that characterize above-ground objects within the urban environment and omit noise artefacts and less expressive features robustly. Such a hypothesis is logical considering the high dimensionality and redundancy generally contained within image data.

We cluster these artificial relief alterations/regularization methods under the name M-ramp (Make a ramp), as ramps are probably the simplest modification one can introduce over an elevation model. To our knowledge, there isn't any previous study considering this kind of artificial relief transformations for delineating features from DEMs, despite the fact that in machine learning literature there is extensive research for constructing transformation invariant feature detectors (Dalal et al., 2005). Even though M-ramp method can be seen as a noisecorruptor applied over an input image, considering the distinct nature of 3D-elevation models, one can strongly experiment with large variations over the input that probably would not make sense over a set of multispectral images. Furthermore, our experiments suggest that a DEM classification could strongly benefit from such artificial relief generation, especially when extended modifications are applied over the input data. The gain from using M-ramp alterations affects the above-ground detection in four distinct ways.

i) Speeds-up model's convergence by a large factor

ii) Helps the model avoid localized, non-regular errors

iii) Increases overall detection accuracy

iv) Generates "new" data

To better understand the impacts and benefits of M-ramp models, we constructed a set of randomly modified DEMs where different types of template-waveforms (with varying magnitudes \& frequencies) were considered along the horizontal and vertical image dimensions. Continuously, these artificially generated DEMs are appended over the initial DEM, creating the final M-ramp models (Figure 1). At this point, it is important to underline that our M-ramp models were randomly designed without conducting any prior study regarding their effects over the accuracy of the above-ground detection problem. Therefore, their positive impact towards our classification results wasn't guaranteed. In addition, it is important to note that the MLP hyperparameters were set using initial untransformed data, hence the MLP is not optimally tuned for these particular M-ramps.

All different construction parameters of the M-ramps DEM models considered in this study are summarized in Table 2 . 


\begin{tabular}{|c|c|c|c|c|}
\hline $\begin{array}{c}\text { Waveform } \\
\text { Type }\end{array}$ & $\begin{array}{c}\text { Magnitude } \\
\text { x-direction } \\
\text { (m) }\end{array}$ & $\begin{array}{c}\text { Frequency } \\
\text { x direction } \\
(\mathbf{H z})\end{array}$ & $\begin{array}{c}\text { Magnitude } \\
\text { y-direction } \\
\text { (m) }\end{array}$ & $\begin{array}{c}\text { Frequency } \\
\text { y-direction } \\
(\mathbf{H z})\end{array}$ \\
\hline Ramp & 0 & - & 100 & - \\
\hline Ramp & 300 & - & 200 & - \\
\hline Ramp & 200 & - & 0 & - \\
\hline Sawtooth & 100 & $8 \cdot 10^{-5}$ & 20 & $5 \cdot 10^{-6}$ \\
\hline Sawtooth & 100 & $8 \cdot 10^{-5}$ & 200 & $7 \cdot 10^{-5}$ \\
\hline Sawtooth & 200 & $8 \cdot 10^{-5}$ & 30 & $6 \cdot 10^{-4}$ \\
\hline Sine & 100 & $3 \cdot 10^{-5}$ & 150 & $5 \cdot 10^{-5}$ \\
\hline Sine & 200 & $9 \cdot 10^{-5}$ & 150 & $10^{-4}$ \\
\hline $\begin{array}{c}\text { Waveform } \\
\text { Type }\end{array}$ & $\begin{array}{c}\text { Noise } \\
\text { magnitude }\end{array}$ & $\begin{array}{l}\text { Gaussian } \\
\text { filtering }\end{array}$ & $\begin{array}{c}\text { Gaussian } \\
\text { std }\end{array}$ & \\
\hline Noise & 150 & No & - & \\
\hline Noise & 5000 & Yes & 15 & \\
\hline Noise & 3000 & Yes & 5 & \\
\hline
\end{tabular}

Table 2. M-ramp waveform construction parameters

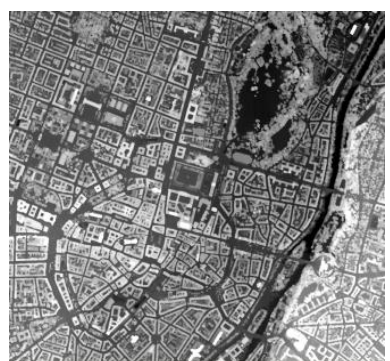

A

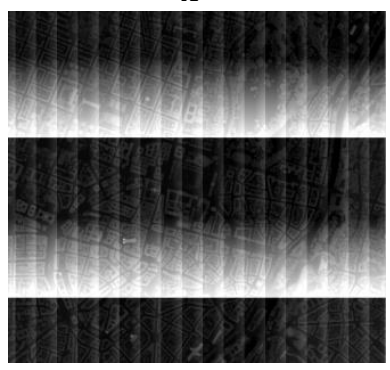

C

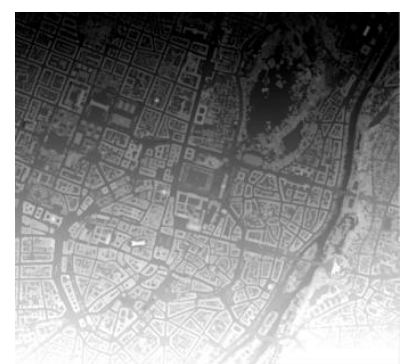

B

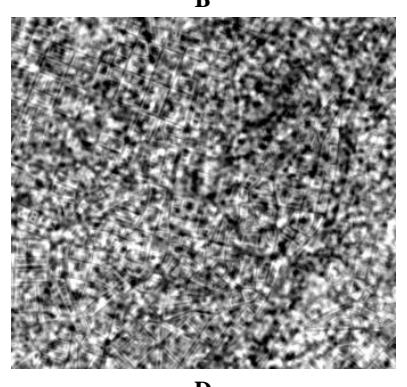

D
Figure 1. Munich center DEM subset with various M-ramp modifications. Image A initial DEM no-modification. Image

$\mathrm{B}$ ramp waveform modification. Images $\mathrm{C}$ two directional sawtooth wave. Image D white-noise modifications

\subsection{Merging Prediction Models}

Model combination is known to nearly always improve the performance of machine learning methods (Srivastava et al., 2014). To tackle this aspect, one can either use multiple different DL architectures and combine their outcomes or change the input data provided to the system and use a standardized classifier. Since finding a proper architecture in DL proves to be a hard task, we decided to use the approach of altering the input data of the MLP classifier. For implementing this, one needs to have a classification outcome that can be combined. Therefore instead of assigning a class-label as predicted outcome, we computed probability-maps which designate the probability of a particular pixel being an aboveground structure. Below we present two indicative ways we considered for merging models without using additional training data.

3.3.1 Altering the Input: Altering the input data of a classifier becomes a trivial process when implementing the previously discussed M-ramp method. In order to proceed with the alteration, one just needs to combine the predictions generated by the individual models trained over the various artificial terrains, constructed by the M-ramp method. This technique provided the MLP model with "new" data which trained the MLP model in distinct ways.

3.3.2 Changing the Contextual Information: For further enhancing the model's prediction accuracy, we constructed two sets of input data-tiles of $15 \times 15$ and $31 \times 31$ pixels respectively. This change in the input size-dimension has a strong impact on the classification outcome as it significantly alters the contextual information provided to the network per class-label. Even though further investigation on this direction is required, initial results suggest that smaller tiles drive the system towards detecting localized information where large tiles force the system to learn more general, larger-scale features.

The two distinct tile-sizes were defined through a logical and visual analysis of the spatial resolution of the scene, in relation to the size of contextual information that would be adequate for differentiating between ground and above-ground through human perception. However, despite our choice upon these particular sizes other values could also be suitable for the task.

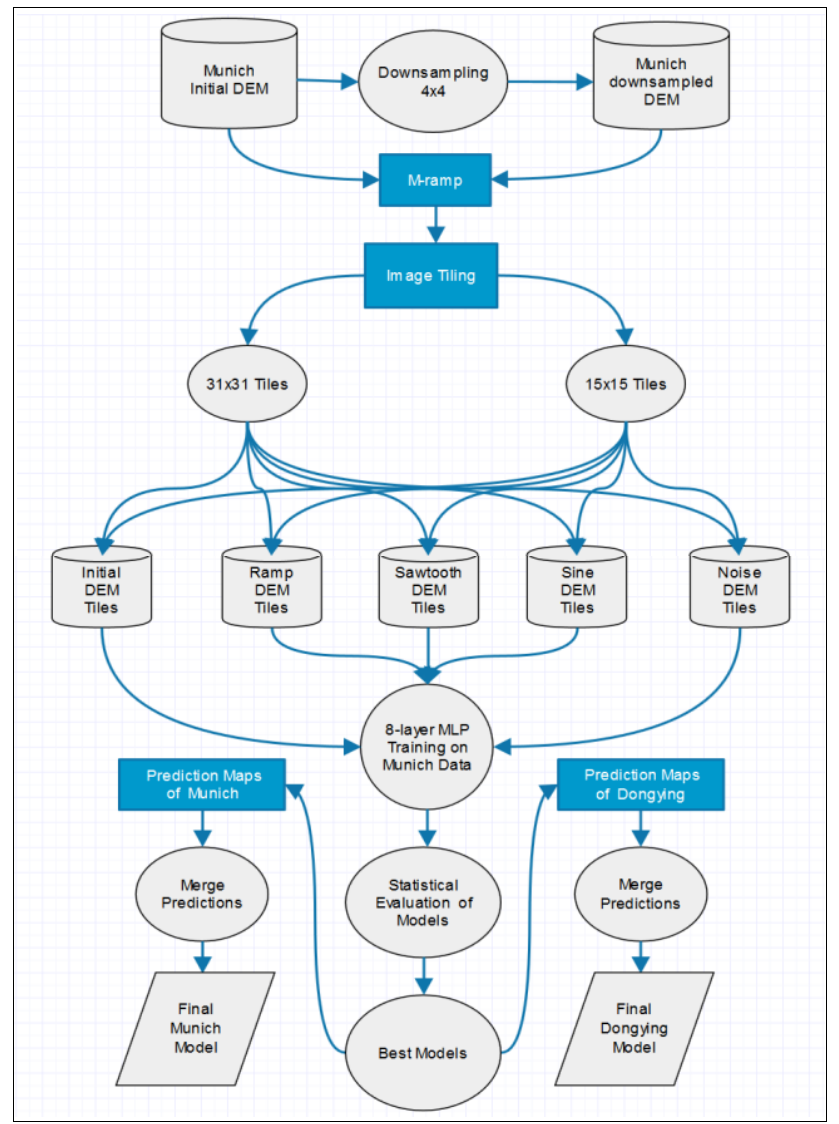

Figure 2. Complete workflow diagram, presenting all procedural steps for above-ground object detection

\subsection{Downsampling DEM Data}

As one of our main goals is to detect the above-ground objects contained within a lower spatial resolution DEM, namely Dongying city, it is necessary to construct a model capable of detecting data in the scale of the prediction-target. One simple approach towards this goal is to train our standardized architecture over a downsampled version of the initial data, as well as some M-ramp variations of it. 
We found the downsampling step to be crucial for predicting data with significantly different resolutions like the ones considered in our experiments with a different scale-factor equal to 16 (Munich $1 \times 1 \mathrm{~m}^{2} /$ pixel, Dongying $4 \times 4 \mathrm{~m}^{2} /$ pixel). However, preliminary experiments suggest that when data with small variations in resolution are considered, the algorithm can predict scenes accurately throughout the different spatial-scales. Nevertheless, the spatial invariance of the method is not investigated further in this study

\section{STUDY AREA}

\subsection{Study Area and Training Challenges}

In our experiments, we used two very heterogeneous DEMs, one delineating the urban and peri-urban area of Munich with an extent of $424 \mathrm{~km}^{2}$ and spatial a resolution of 1-meter, as well as a DEM of Dongying city in China, extending over an $257 \mathrm{~km}^{2}$ with a spatial resolution of 4-meters. Our hypothesis is that a trained Deep Neural Network will be able to correctly detect all above-ground structures in both Dongying and Munich datasets under the following two-constrains:

i) Limit potential training area to a small subset of Munich with an extent of $25 \mathrm{~km}^{2}$

ii) Only allow the classifier to sample 20000 random single pixel-labels from this subset and use it for training (comprise $\sim 0.08 \%$ of Munich subset or $\sim 0.005 \%$ of complete Munich data)

The Munich subset we selected depicts the city center of Munich (extent $\sim 25 \mathrm{~km}^{2}$ ). Additionally, for detecting the aboveground objects in Dongying dataset, we trained the very same MLP model over a downsampled version of the Munich subset (downsampled to 4-meters), using again 20000 randomly selected point-samples.

\subsection{Information on DEMs}

The first dataset of this study is a VHSR DEM produced from optical stereotypical, aerial images with a ground resolution of 1-meter. This data covers the greater area of Munich with an overall extent of $424 \mathrm{~km}^{2}$ (22800 x 18600 pixels). Additionally, in order to test our prediction model over a different urban landscape, a second DEM of 4-meters resolution was also considered. The latter was generated from a stereopair of Pleiades-sensor optical images and depicts the complete extent of Dongying city, located in Yellow River Delta, China. The Dongying dataset covers an area of about $257 \mathrm{~km}^{2}$ and has very different architectural characteristics compared to the Munich dataset. Precisely, apart from the different resolution of the data which significantly affects the level of details, the Munich dataset is rather elevated with about 100 meter height difference between its north and south side. Comparatively, the Dongying dataset is very flat, with minimal changes in ground-elevation. Furthermore, noticeable differences occur with respect to the density, shapes and sizes of the building structures between this dataset, as both resemble two very different city models. For instance, Munich's structures are homogenously distributed with an overall low height-level where Dongying's buildings are higher and sparsely located, with periodic dense concentrations.

\subsection{Above-Ground Labels}

Since the datasets used in this study are quite large, obtaining a ground truth necessary for the training and statistical evaluation of the model was a challenging task (Munich city center subset
\& complete Dongying city). Therefore, a reasonable solution was to use another algorithm to generate the above-ground labels. To this aim we employed a method we have previously proposed (Marnoncini et al., 2014) which was constructed to deal with medium resolution DEM data, acquired by the TanDEM-X/ TerraSAR-X mission. However, due to the fact that this algorithm was not designed to deal with VHSR data, some manual post-editing processing was found necessary. Furthermore, due to some residual mistakes, we estimate that we still have an error in our ground-truth data of about $\sim 7 \%$ over all of our label-data.

Figure 3 depicts the DEM of an area in Munich along with its' labelled map. It is clear that the labels have a considerable amount of errors which results in some statistical inaccuracies. By implementing manual editing, many of the errors were corrected, however due to the large extent of the data, a detailed correction on a structure-by-structure basis was not feasible.
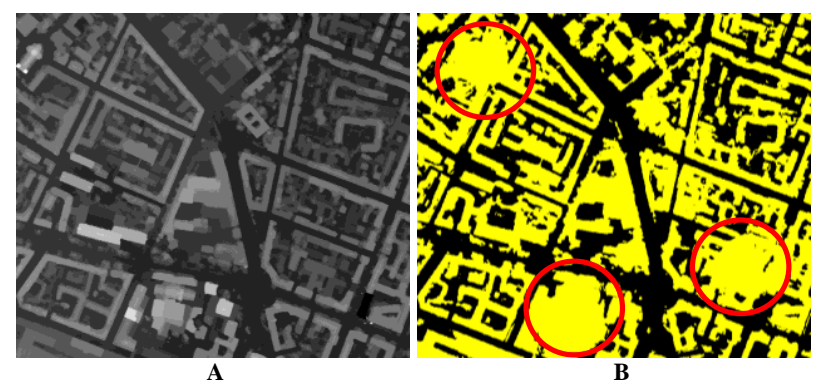

Figure 3. Small extent of Munich city center dataset. Image A depicts the initial DEM where image $B$ the respective ground-truth labels, containing some significant errors

\section{EXPERIMENTS}

\subsection{Experiments and Accuracy Assessment}

In contrast to highly-specialized algorithms which can only be applied over small data sets containing few buildings (computational constraints), our model is capable of detecting above-ground structures over very large extents - in just a few minutes, robustly. For supporting this statement we quantitatively assessed our method's performance, considering a strict error measurement on pixel-by-pixel basis using confusion matrices. Statistical results were calculated over the subset of Munich city-center and the complete extent of Dongying-city. The only post-processing step considered (prior to the statistical evaluation) was a filtering of the prediction map with a $5 \times 5$ pixel-size median filter (compensate for localized false positives errors). In the rest of this section, we present the different considered experiments and their respective results.

\subsection{Training the Flat-Model}

In our experiments, when we refer to the term "Flat-model" we indicate using the initial DEM with no M-ramp alterations as an input to the MLP. Hence, in our first experiment, we only considered this flat-model over the Munich center dataset. Interestingly, the results reflected that we can achieve high detection performance even with this limited model. Statistical and visual results can be found in Figure 4 and Table 3 respectively. 


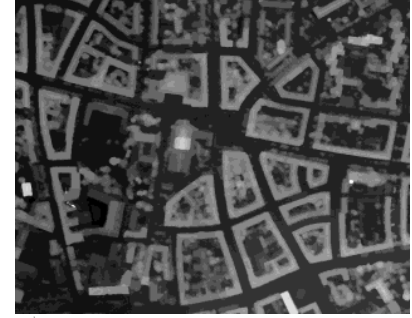

A

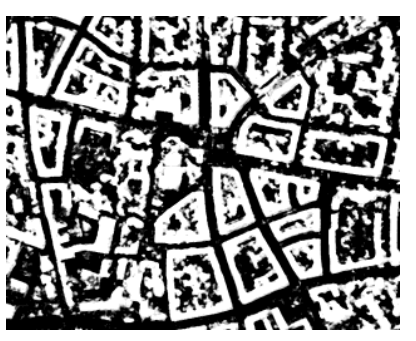

B
Figure 4. Small area of the initial Munich DEM. Image A illustrates initial DEM. Image B depicts respective prediction map with tiles of size $31 \times 31$, Flat-model

\begin{tabular}{lcc}
\hline \hline $\begin{array}{c}\text { Model Munich City } \\
\text { Center }\end{array}$ & $\begin{array}{c}\text { Above- } \\
\text { ground [\%] }\end{array}$ & $\begin{array}{c}\text { Ground } \\
{[\%]}\end{array}$ \\
\hline \hline Flat-model, 15X15 tiles & 86.3 & 85.6 \\
Flat-model, 31X31 tiles & 86.6 & 88.6 \\
Ramp-3, 15X15 tiles & 88.3 & 83.1 \\
Ramp-3, 31X31 tiles & 87.5 & 87.7 \\
Best four M-ramps merged & $\mathbf{8 9 . 7}$ & $\mathbf{8 9 . 1}$ \\
\hline \hline \multirow{2}{*}{ Model Dongying City } & Above- & Ground \\
& ground [\%] & {$[\%]$} \\
\hline \hline Flat-model, 15X15 tiles & 89.1 & $\mathbf{8 9 . 7}$ \\
Flat-model, 31X31 tiles & 72.8 & 85.3 \\
Ramp-3, 15X15 tiles & 85.1 & 76.2 \\
Ramp-3, 31X31 tiles & 87.2 & 83.3 \\
Best four M-ramps merged & $\mathbf{8 9 . 7}$ & 89.3 \\
\hline \hline
\end{tabular}

Table 3. Prediction accuracy of all investigated models for Above-Ground \& Ground classes (pixel-by-pixel).

\subsection{Training on M-ramp Models}

In order to increase our prediction accuracy, we continuously employed the previously discussed M-ramp method. Figure 5 shows the error rates throughout training epochs over the various M-ramp models (formerly constructed), over a test set. It's important to observe in the graph (Figure 5) that most of the M-ramp models achieve lower error-rate (higher accuracy) than the initial flat model and furthermore converge significantly faster. Detailed information regarding the gains in respect to the use of the various M-ramps are summarized in the Table 4. A visual result is given in Figure 6.

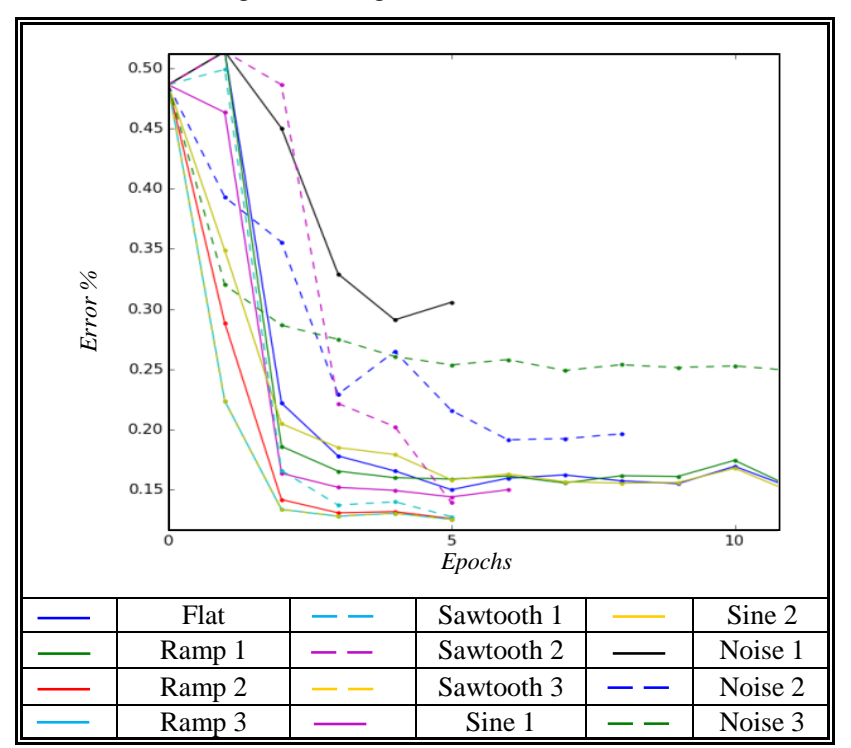

Figure 5. Error rates of different M-ramp 31x31 models during training epochs over a test-dataset

\begin{tabular}{ccc}
\hline \hline M-ramp Models & $\begin{array}{c}\text { Convergence } \\
\text { Epoch }\end{array}$ & $\begin{array}{c}\text { Test Set } \\
\text { Error [\%] }\end{array}$ \\
\hline \hline Flat & 41 & 14.8 \\
\hline \hline Ramp-1 & 72 & 14.9 \\
Ramp-2 & 9 & 12.6 \\
Ramp-3 & $\mathbf{5}$ & $\mathbf{1 2 . 5}$ \\
\hline \hline Sawtooth-1 & 5 & 12.8 \\
Sawtooth-2 & 5 & 13.9 \\
Sawtooth-3 & $\mathbf{5}$ & $\mathbf{1 2 . 5}$ \\
\hline \hline Sine -1 & 6 & 14.9 \\
Sine-2 & 41 & 15.1 \\
\hline \hline Noise-1 & 5 & 32.0 \\
Noise-2 & 8 & 19.5 \\
Noise -3 & 56 & 22.0 \\
\hline \hline
\end{tabular}

Table 4. Detailed results of various M-ramps on convergence time and error rates (test-set). Results are calculated over $31 \times 31$ data-tiles
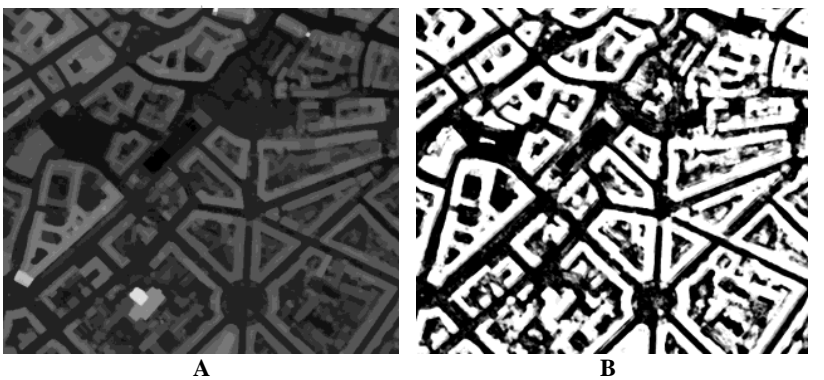

Figure 6. Subset of Munich city center dataset. Image A initial DEM. Image B prediction map trained over M-ramp, Ramp-3 model

\subsection{Varying the Input Dimension}

In our experiments, as an input-dimension to the MLP, we have considered two separate tile-sizes. Precisely, tests were conducted over $31 \times 31$ and $15 \times 15$ pixel-size tiles and statistically evaluated (Table 3). This additional step was included due to non-systematic, localized errors, occurring when different tile-sizes were considered (due to the minimal training dataset). Despite the different tile-sizes, the models overall agree in their predictions, nonetheless little difference occur over limited areas throughout the scene (low tree vegetation, large bushes, cars etc.) Furthermore, we may argue that both tile-sizes were found to produce accurate results and their outcomes can be considered complementary and nonredundant.

\subsection{Merging Top Performance M-ramp Models}

Even though most of the individual M-ramp models improved the overall accuracy of above-ground detection, localized errors still occur in the individual prediction maps. As these errors were found to be non-systematic throughout the various models, a good solution was to merge the individual prediction maps. This averaging process enforces the detection over structures that are repeatedly detected correctly and cancels out the individual-localized errors which exist only in particular models. This hypothesis is further supported by the statistical results of the discussed merged-model where the top four $\mathrm{M}$ ramp models \{15x15 Ramp-3, 31x31 Ramp-3, 15x15 Sawtooth$3 \& 31 \times 31$ Sawtooth-3\} produced the best outcome with an accuracy of approximately $90 \% \quad(24.8 \%$ relative-error improvement in relation to Flat model). The improvements are displayed in Table 3 and Figure 7 and 8 respectively. 


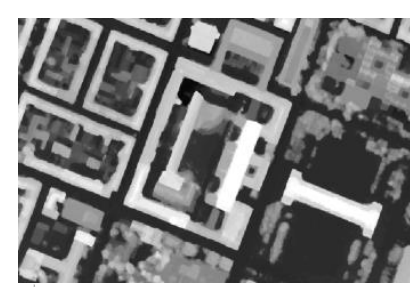

A

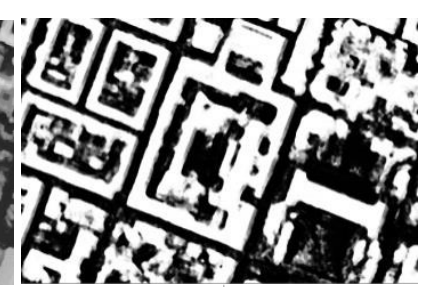

Figure 7. TUM test-area. Image A initial DEM model. Image B Merged M-ramp prediction map
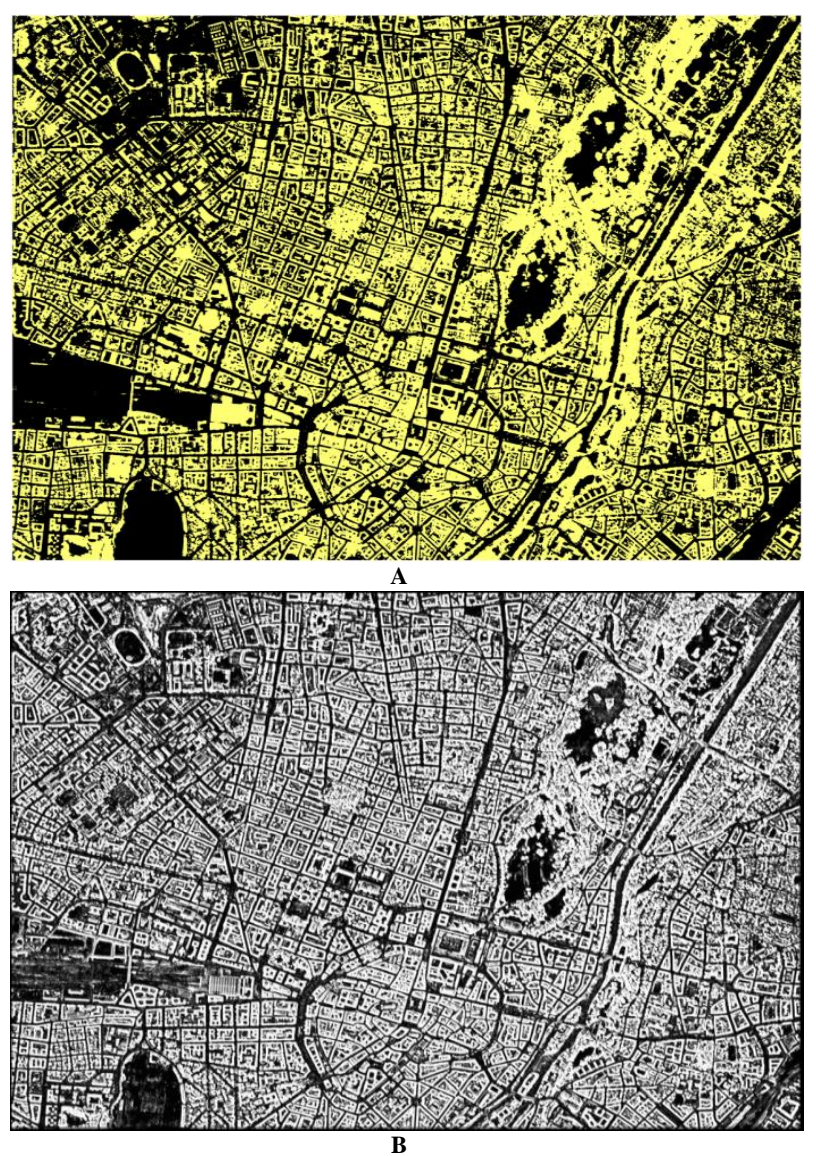

Figure 8. Complete Munich city center test area. Image A depicts label / ground-thruth data. Image B illustrates the computed Merged prediction map

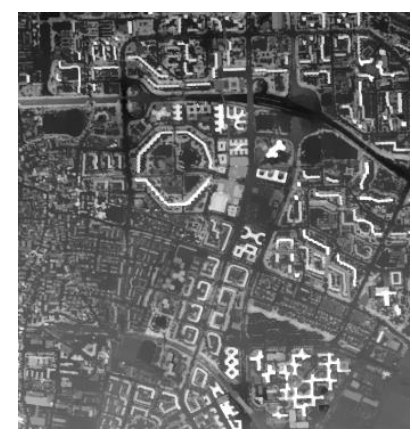

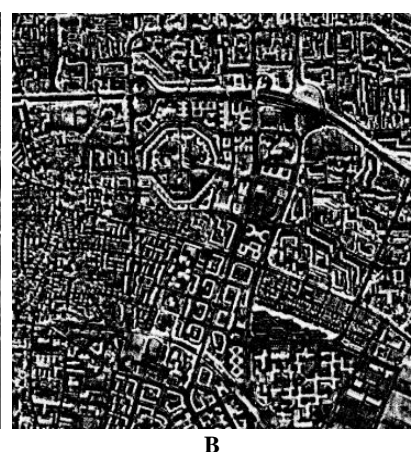

Figure 9. Prediction over unseen extent from the greater area of Munich. Image A depicts the initial DEM. Image B pictures the predicted above-gournd 31x31, Ramp-3 map

\subsection{Predicting the Greater Munich Area}

One important step towards proving transferability of the MLP was the prediction of the greater unseen Munich dataset. This encapsulates the complete Munich city in addition to various neighbouring suburbs, agricultural fields, forests and lakes lying around the core city. For this larger extent, a ground-truth dataset was not available therefore, only a qualitative assessment was feasible. Nevertheless, throughout this area, the algorithm achieved great visual performance, as can be seen in Figure 9, where a large area is accurately separated in above-ground and ground classes.

\subsection{Training Over Munich - Predicting over Dongying}

As a challenging experiment towards model-transferability, we performed yet another classification over a very diverse dataset, without providing any insight to the classifier regarding this urban DEM. The only necessary step was to retrain our standardized top-four performing M-ramps models over a downsampled version of Munich-center dataset. This allowed the individual datasets to acquire same spatial resolutions (4meters) so the classifier can adapt on a new level of spatial detail. Final results were again considered after the merging of the individual M-ramp models, as in the Munich-subset study. The quantitative evaluation was computed over the complete extent of the Dongying DEM, on a pixel-by-pixel basis. In this case the algorithm again achieved high performance of about $90 \%$ (5.5\% relative-error improvement in relation to Flat model). Statistical and visual results of this experiment can be found in Table 3 and Figure 10 respectively.
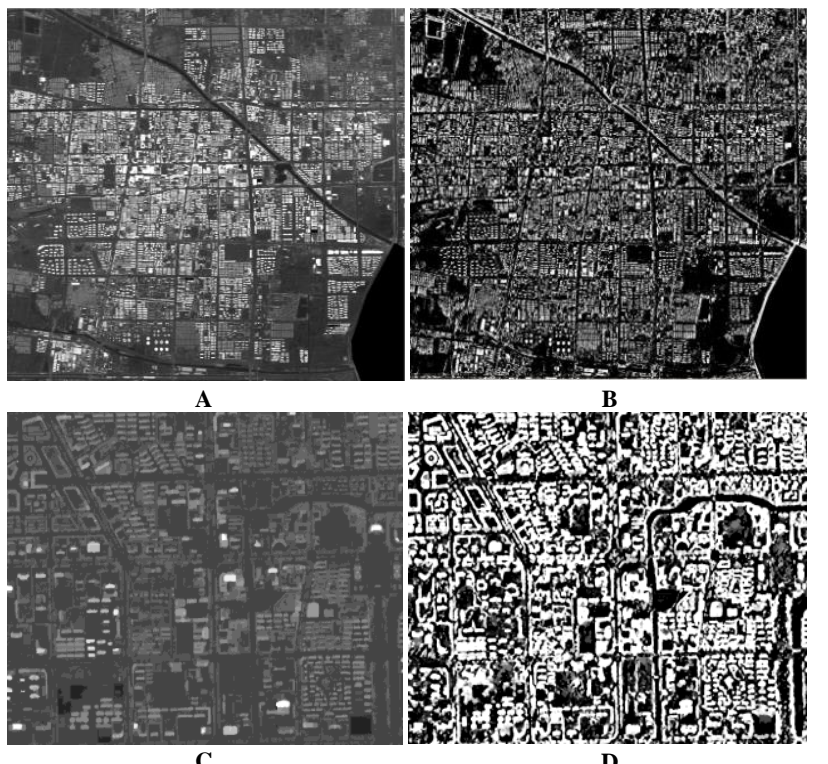

Figure 10. Prediction over unseen Dongying city, China.

Image $\mathrm{A}$ and $\mathrm{C}$ depict different parts of the initial DEM where images B and D contain the respective prediction maps

\section{DISCUSSION \& SUMMARY}

\subsection{Computational Efficiency \& Model Reusability}

One strong advantage of the proposed method pertains to the computational efficiency of the algorithmic model. Precisely, due to the difficulty of defining the hyperparameters of the MLP, the algorithm requires quite some time to be constructed. However, once the model is composed, the actual detection of 
the above-ground objects concludes in just a few minutes. Furthermore, through a series of experiments, we provided evidence regarding the transferability of the model without the need of re-training the system (for data with same spatial resolution). This key aspect further supports the potential reusability of DL classifiers in the context of above-ground detection.

\subsection{Remarks on M-ramp}

Even though our experiments suggest that M-ramp can generally improve the above-ground object detection within the urban extent, it is important for one to understand the limitations imposed by the classification algorithm prior to using it. M-ramp can improve DEM classification as long as the algorithm has a large enough capacity to accurately detect and model all variations contained within the training data and still have enough potential left to compensate for the information introduced by the M-ramp method. Therefore providing extensively altered relief to a strongly restricted / regularized classifier may result in a decrease in classification accuracy. Such a case is visible in Figure 5 where the white-noise models clearly drop in overall accuracy. Nonetheless, despite this decline the majority of the other M-ramps significantly enhanced model correctness and convergence time. All the above facts establish M-ramp as an additional regularizer in the context of DL and DEM classification and suggests that their engaging properties should be further investigated in future studies.

\subsection{Remarks over Classification Accuracies}

Even though the accuracies we have acquired in the task of above-ground object detection were quite high, we strongly believe that the actual predictions are more precise than statistically presented. Considering the error rate of the labels lying around $7 \%$, one can understand the significant impact they result over our final error estimation. This argument can be further supported by visual comparison where the prediction maps state the truth and the labels are wrong. In addition, a separation in ground and above-ground classes over a label/ground-truth dataset is a non-trivial and quite subjective task due to the existence of multiple objects that could potentially belong to either class (bushes, cars, large rock formations, etc.)

\subsection{Summary}

In this work, we presented a generic framework for aboveground object detection using MLP over a raw DEM model. We have showed that even by extensively shrinking the training dataset, we can still achieve very good performance and even re-use the classifier over different unseen datasets. Additionally, we introduced a new method, called M-ramp which significantly improves performance and convergence time. These aspects clearly highlight the potentials of DL in the field of remote sensing and we hope that our work will inspire researchers in the field to implement these techniques to accomplish new milestones.

As future implementations of DL in DEM object extraction, we would be interested in trying to separate between buildingstructures and vegetation by again using raw DEM data. Additionally, in the same context, the implementation of different DL models such as Convolutional Neural Networks and Deep Belief Network would be also of great interest.

\section{REFERENCES}

Baillard, C., Matre, H., 1999. 3D reconstruction of urban scenes from aerial stereo imagery: a focusing strategy. Computer Vision and Image Understanding, 76(3), pp. 244-258.

Belli, T., Cord, M., Jordan, M., 2001. 3D Data reconstruction and modeling for urban scene analysis. In Workshop of Automatic Extraction of Man-made Objects from Aerial and Satellite Images, A.A. Balkema Publishers, (3), pp. 125-134.

Dalal, N., Triggs, B., 2005. Histograms of oriented gradients for human detection. In IEEE Conference on Computer Vision and Pattern Recognition 2005, (1), pp. 886-893.

Marconcini, M., Marmanis, D., Esch, T., Felbier, A., 2014. A novel method for building height estimation using TanDEM-X data. In IEEE International Geoscience and Remote Sensing Symposium (IGARSS), 2014, pp. 4804-4807.

Ortner, M., Descombes, X., Zerubia, J., 2007. Building outline extraction from digital elevation models using marked point processes. International Journal of Computer Vision, 72(2), pp. 107-132.

Rosenblatt, F., 1957. The Perceptron - a perceiving and recognizing automaton. Report 85-460-1, Cornell Aeronautical Laboratory.

Rumelhart, D., Hinton, G., Williams, R., 1986. Learning representations by back-propagating errors. Nature 323, pp. 533-536.

Sirmacek, B., \& Unsalan, C., 2009. Urban-area and building detection using SIFT keypoints and graph theory. In IEEE Transactions on Geoscience and Remote Sensing, 47(4), pp. 1156-1167.

Srebro, N., Shraibman A., 2005. Rank, trace-norm and maxnorm. In Proceedings of the 18th annual conference on Learning Theory (COLT) 2005, pp. 545-560.

Srivastava, N., Hinton, G., Krizhevsky, A., Sutskever, I., Salakhutdinov, R., 2014. Dropout: A simple way to prevent neural networks from overfitting. The Journal of Machine Learning Research, 15(1), pp. 1929-1958.

Tournaire, O., Bredif, M., Boldo, D., Durupt, M., 2010. An efficient stochastic approach for building footprint extraction from digital elevation models. ISPRS Journal of Photogrammetry and Remote Sensing, 65(4), pp. 317-327.

Vincent, P., Larochelle, H., Bengio, Y., Manzagol, P., 2008. Extracting and composing robust features with denoising autoencoders. In Proceedings of the 25th International Conference on Machine Learning, pp. 1096-1103.

Zingaretti, P., Frontoni, E., Forlani, G., Nardinocchi, C., 2007. Automatic extraction of LIDAR data classification rules. In 14th International Conference on Image Analysis and Processing (ICIAP) 2007, pp. 273-278. 